\title{
Comportamento tensão-deformação-resistência de uma argila orgânica mole da região norte da cidade do Recife-PE, Brasil
}

Stress-deformation-resistance behavior of soft organic clay from the northern region of the city of

Recife-PE, Brazil

Comportamiento tensión-deformación-resistencia de una arcilla orgánica blanda de la región norte de la ciudad de Recife-PE, Brasil

Recebido: 24/01/2022 | Revisado: 29/01/2022 | Aceito: 05/02/2022 | Publicado: 10/02/2022

\author{
Sílvio Romero de Melo Ferreira \\ ORCID: https://orcid.org/0000-0002-5760-1494 \\ Universidade Federal de Pernambuco, Brasil \\ E-mail: silvio.mferreira@ufpe.br \\ Joaquim Teodoro Romão de Oliveira \\ ORCID: https://orcid.org/ 0000-0001-8361-2296 \\ Universidade Federal de Pernambuco, Brasil \\ Universidade Católica de Pernambuco, Brasil \\ E-mail: jtrdo@uol.com.br \\ Ana Nery Cadete \\ ORCID: https://orcid.org/0000-0001-8135-0288 \\ Universidade Federal de Pernambuco, Brasil \\ E-mail: aninhacadete@yahoo.com.br \\ Felipe Melo de Assis Rocha \\ ORCID: https://orcid.org/0000-0002-9974-4848 \\ Universidade Católica de Pernambuco \\ E-mail: feliperocha.eng@hotmail.com \\ Manoely Souza de Oliveira \\ ORCID: https://orcid.org/0000-0002-6996-8082 \\ Universidade Federal de Pernambuco, Brasil \\ Instituto Federal da Paraiba, Brasil \\ E-mail: manoely.oliveira@hotmail.com
}

\begin{abstract}
Resumo
Depósitos sedimentares de solos moles, ocorrem na costa brasileira. Apresentam elevado conteúdo de matéria orgânica, alta compressibilidade, baixa permeabilidade e resistência ao cisalhamento. São solos problemáticos para o uso e ocupação. A planície de origem fluvio-marinha da cidade do Recife apresenta mais de $50 \%$ de sua área composta por solos moles. $\mathrm{O}$ artigo avalia o comportamento tensão-compressão-resistência da argila orgânica mole localizada na região Norte do Recife-PE, Brasil. Em campo, foram coletadas amostras indeformadas, realizados ensaios de Piezocone e Vane Test. Em laboratório, foram realizados ensaios de compressão unidimensional com drenagem vertical e horizontal e triaxial. A secagem prévia é analisada na determinação dos limites de liquidez e plasticidade, a qualidade das amostras e a história de tensão são também analisadas. O perfil do solo apresenta uma camada superficial de areia siltosa e silte arenoso seguido de camadas de aluviões muito moles com presença de turfa e argila, de baixa resistência e alta compressibilidade. A argila é levemente pré-adensada até $4,0 \mathrm{~m}$ de profundidade e a partir desta profundidade, é normalmente adensada. A relação entre o coeficiente de adensamento horizontal e o vertical é 3,70. Há certo amolgamento nas amostras do solo. A resistência não drenada média nos $4 \mathrm{~m}$ iniciais é 35 $\mathrm{kPa}$ e, em profundidades maiores, $25 \mathrm{kPa}$. A argila é mais sensível nos $4,0 \mathrm{~m}$ iniciais. A resistência não drenada obtida por ensaios de laboratório (UU) são próximas às obtidas por ensaios de vane de pico.
\end{abstract}

Palavras-chave: Argilas moles; Parâmetros de compressibilidade; Qualidade das amostras; Resistência não drenada.

\begin{abstract}
Sedimentary deposits of soft soils occur on the Brazilian coast. These soils usually have high organic matter content, high compressibility, low permeability and shear resistance. They are problematic soils for use and occupation. The alluvial plain of marine fluvial origin in the city of Recife, presents more than $50 \%$ of its area with soft clays. The article evaluates the stress-strain-resistance behavior of soft organic clay located in the northern region of the city of Recife-PE, Brazil. In the field, undisturbed samples were collected and Piezocone and Vane tests were performed. In the laboratory, consolidation tests were carried out with vertical and horizontal drainage and triaxial. The influence of
\end{abstract}


pre-drying in determining the consistency limits was analyzed and quality of the samples at pre-consolidation stress. The soil profile presents a superficial layer of silty sand and sandy silt followed by layers of very soft alluviums with the presence of peat and clay, of low resistance and high compressibility. The clay is slightly pre-compacted to a depth of $4.0 \mathrm{~m}$ and, after this depth, it is normally compacted. The relationship between the horizontal and vertical densification coefficient is 3.70. The samples show some dents. The average undrained resistance in the initial $4 \mathrm{~m}$ is $35 \mathrm{kPa}$ and, in depth greater than $25 \mathrm{kPa}$. Clay is more sensitive in the initial $4.0 \mathrm{~m}$. The undrained resistances obtained by laboratory tests (UU) are close to those obtained by peak vane tests.

Keywords: Soft clays; Compressibility parameters; Sample quality; Undrained strength.

\section{Resumen}

Depósitos sedimentarios de suelos blandos ocurren en la costa brasileña. Tienen un alto contenido de materia orgánica, alta compresibilidad, baja permeabilidad y resistencia al corte. Son suelos problemáticos para el uso y ocupación. La llanura aluvial de origen fluviomarino de la ciudad de Recife, presenta más del $50 \%$ de su área compuesta por suelos blandos. El artículo evalúa el comportamiento tensión-deformación-resistencia de una arcilla orgánica blanda ubicada en la región norte de la ciudad de Recife-PE, Brasil. En campo, se colectaron muestras no perturbadas y se realizaron pruebas de Piezocone y Vane. En laboratorio se realizaron pruebas de consolidación con drenaje vertical y horizontal y triaxial. Se analiza la influencia del secado previo en la determinación de los límites de consistencia y calidad de las muestras en el tensión de preconsolidación. El perfil del suelo presenta una capa de arena limosa y limo arenoso seguida de capas de aluvión muy blando con presencia de turba y arcilla, de baja resistencia y alta compresibilidad. La arcilla está ligeramente precompactada hasta una profundidad de 4,0 m, a partir de esta profundidad, se compacta normalmente. La relación entre el coeficiente de densificación horizontal y vertical es de 3,70. La resistencia media no drenada en los $4 \mathrm{~m}$ iniciales es de $35 \mathrm{kPa}$, a mayor profundidad, de $25 \mathrm{kPa}$. La arcilla es más sensible en los 4,0 m iniciales. Las resistencias no drenadas obtenidas por las pruebas de laboratorio (UU) se aproximan a las obtenidas por las pruebas de pico de Vane.

Palabras clave: Arcillas blandas; Parámetros de compresibilidad; Calidad de la muestra; Resistencia no drenada.

\section{Introdução}

Depósitos de argilas moles ocorrem em todo mundo e constituem um desafio à Engenharia Civil. No Brasil são encontrados em várias cidades como na Baixada Santista-SP (Massad, 2009), no Rio de Janeiro (Almeida et al, 2008), em Porto Alegre-RS (Bressani et al, 2011), em Vitória-ES (Santos et al, 2018) em Sergipe (Sandroni.et al, 1997) e em Belém-PA, as argilas orgânicas de baixa resistência ocorrem em áreas de várzea, Vieira et al (2021). No Recife, as argilas moles vêm sendo estudadas há mais de 60 anos. Os estudos tiveram início com o trabalho de Costa (1960) sobre o subsolo do Recife. Teixeira (1972) estudou a influência da matéria orgânica em três perfis geotécnicos que ilustram a ocorrência da matéria orgânica. Amorim Jr. (1975) estudou as características de compressibilidade e resistência da argila mole no bairro da Madalena. Soares (1975) e Carvalho (1975) estudaram a argila no bairro do Derby. Seguiram essa linha de caracterização geotécnica das argilas moles Coutinho (1980), Ferreira (1982), Ferreira, Amorim Jr \& Coutinho (1986), Oliveira (1991 e 2002), Coutinho, Oliveira \& Danziger (1993) e Oliveira (2000) investigando a argila do bairro da Madalena. Ferreira, Amorim e Coutinho (1986) fizeram um estudo pioneiro de banco de dados de solos moles da cidade do Recife. Bello (2011) e Bello et al (2019) estudaram os parâmetros geotécnicos das argilas moles de Suape na região Metropolitana do Recife, Rocha (2016) e Cadete (2016) estudaram as argilas moles na região norte da cidade e Magalhães et al, (2020) investigaram a argila do bairro de Dois Irmãos.

Mais de cinquenta por cento da área da cidade do Recife é formada por depósito resultante do acúmulo de sedimentos trazidos, principalmente, pela ação do mar e dos rios com argila mole contendo matéria orgânica decomposta ou em decomposição, apresentando-se, ora em superfície, ora em profundidade, Ferreira (1982). A planície é limitada pelo mar e pelas Formações Barreiras. O lençol freático encontra-se geralmente próximo à superfície do terreno e as argilas encontram-se normalmente saturadas, Texeira (1972). Oliveira et al (2016) compilou cento e oitenta e oito perfis de solos localizados em vinte e um bairros da planície do Recife, foram grupos em quatro perfis típicos com subgrupos totalizando 9 unidades, onde há intercalação de camadas de argilas orgânicas e inorgânicas de cores e consistência variadas, areias podendo haver, entre estas, 
camadas de siltes, turfas, arenitos e pedregulho. Mais de 50\% dos solos da planície da cidade é constituído por argila, das quais aproximadamente $63 \%$ corresponde àquelas que possuem índice de resistência à penetração (NSPT) $\leq 4$ golpes/0,30 m.

A expansão urbana das cidades, a alta valorização dos terrenos, a construção de estradas, vias de acesso, conjuntos habitacionais, aterros em depósitos de solos moles leva a frequente execução de obras de porte sobre espessas camadas de argilas orgânicas trazendo sérios problemas para a Engenharia Geotécnica devido a alta compressibilidade, baixa capacidade de suporte e baixa permeabilidade desses solos. O desenvolvimento da cidade do Recife levou a elaboração em 2014 de projeto viário na comunidade Chão de Estrelas na região Norte da cidade, Figura 1. A construção da via tem 1,4 km na marginal direita do Rio Beberibe, com 7,0 m de largura para tráfego de veículos, além de ciclovia e passeio com 2,5 m de largura cada. Alguns trechos da via passam por um depósito de solo mole que requer a construção de aterro. Para o dimensionamento do aterro foi elaborado um programa de investigação do solo de fundação para análise da compressibilidade e de resistência ao cisalhamento. Os dados do projeto deram subsídio a duas dissertações. O artigo tem por objetivo analisar de forma integrada a compressibilidade e a resistência ao cisalhamento da argila considerando as investigações geotécnicas realizadas por Rocha (2016) quanto à compressibilidade e por Cadete (2016) quanto à resistência, em suas dissertações.

\section{Metodologia}

O local dos estudos experimentais está inserido em um depósíto de origem fluvio-marinha. A investigação geotécnica em campo foi realizada na comunidade Chão de Estrelas-PE na zona norte da capital pernambucana, próxima aos bairros Campina do Barreto, Arruda e Beberibe. A Figura 1 mostra o local onde foram coletadas as amostras e realizados os ensaios de "vane" e piezocone. A investigação geotécnica mais detalhada do projeto foi concentrada em dois locais, chamados de ilhas de investigação, Figura1. Na Ilha 1, as amostras indeformadas do tipo Shelby foram coletadas nas profundidades de $2 \mathrm{~m}, 8 \mathrm{~m}$ e 11 m, e na Ilha 2, nas profundidades de $2 \mathrm{~m}$ e $8 \mathrm{~m}$. O perfil geotécnico do solo da Ilha 1 (SPT-203-A) até a Ilha 2 (SPT-208-A) é mostrado na Figura 2. A Tabela 1 apresenta as porcentagens dos grãos dos solos da Ilha 1 e da Ilha 2, provenientes da investigação geotécnica realizada por Cadete (2016).

Figura 1 - Localização das ilhas de investigação.

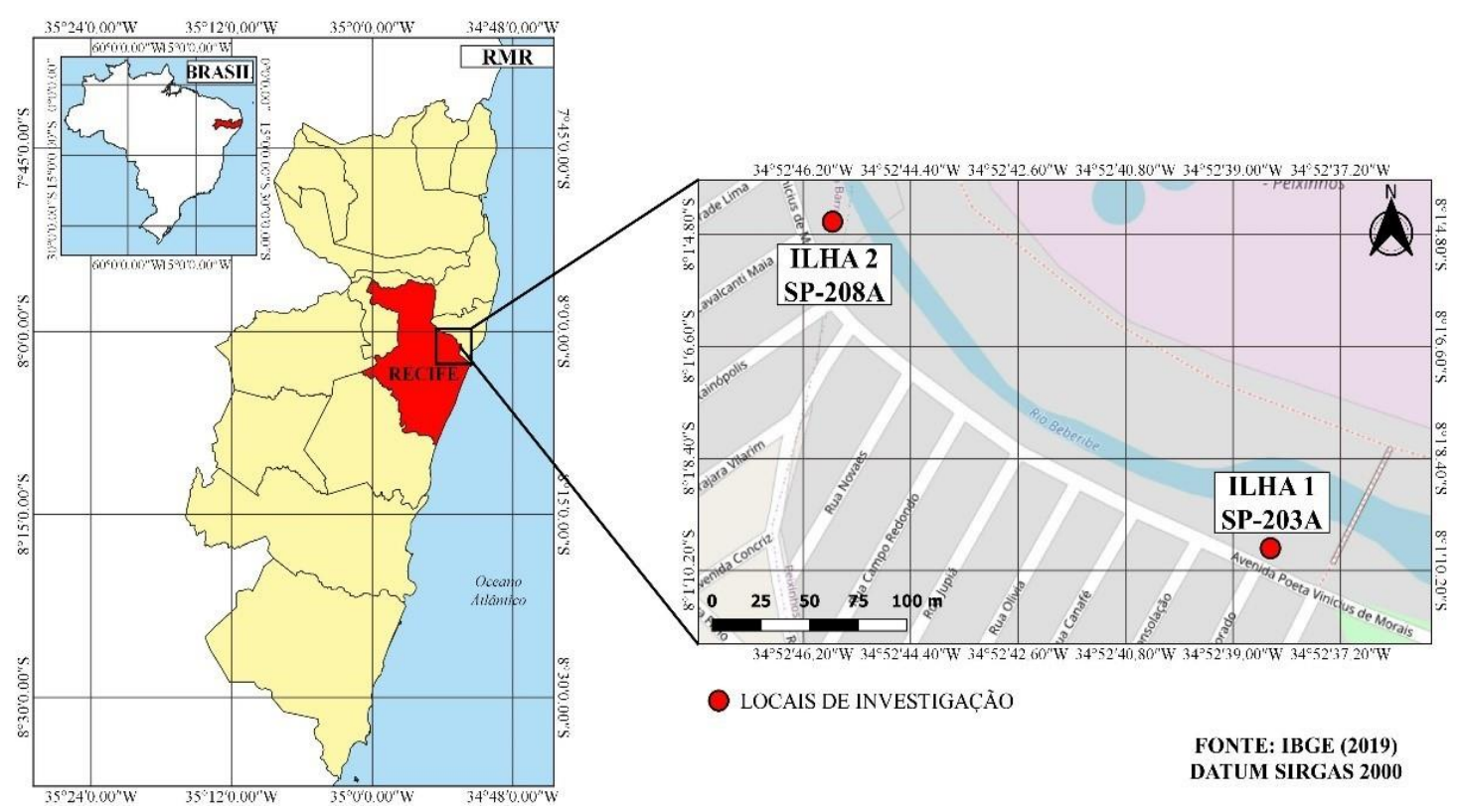

Fonte: Autores. 
Figura 2 - Perfil Geotécnico desde a Ilha 1 (SPT-203-A) até a Ilha 2 (SPT-208-A).

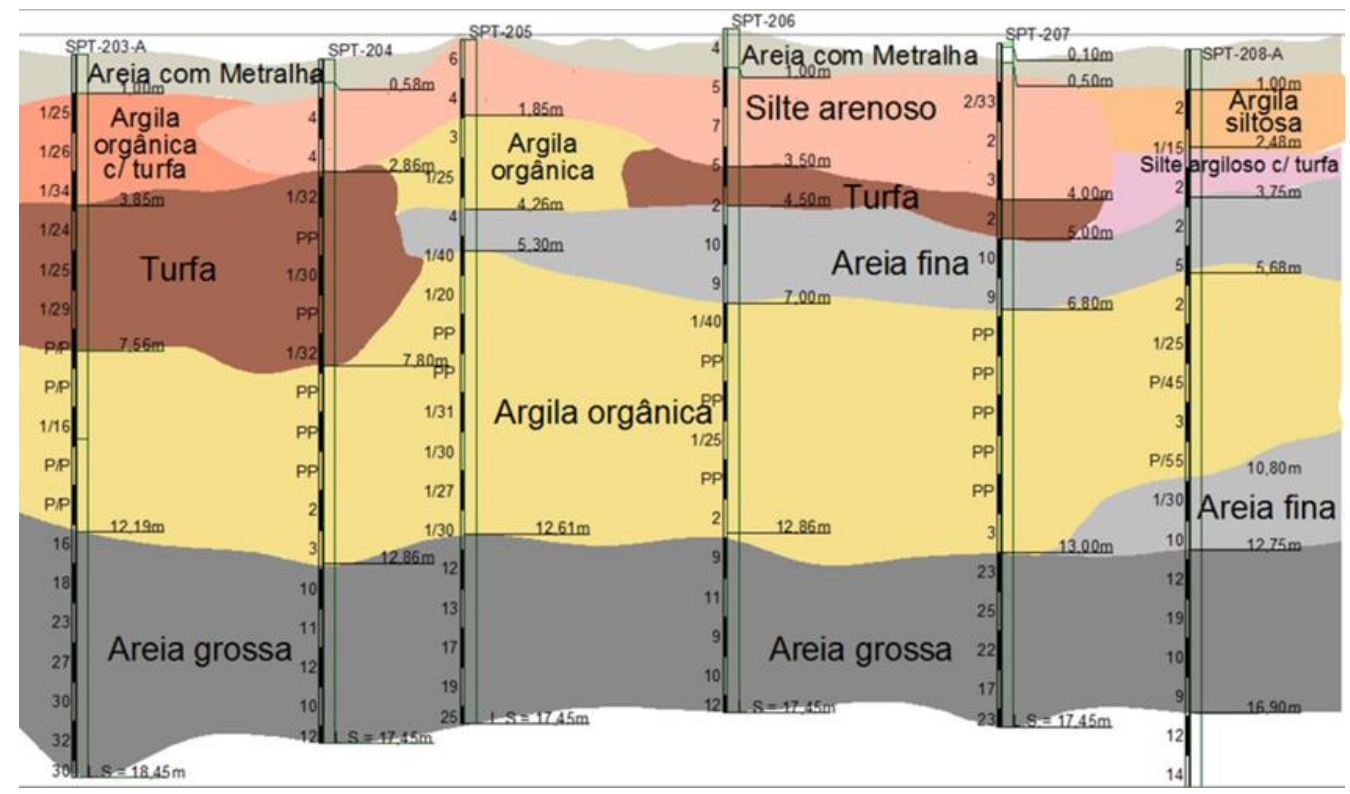

Fonte: Autores.

Tabela 1 - Resultado da Granulometria.

\begin{tabular}{cccccc}
\hline Local & Prof. (m) & Areia média (\%) & Areia fina (\%) & Silte (\%) & Argila (\%) \\
\hline Ilha - 1 & 2,00 & 10 & 37 & 16 & 37 \\
Ilha - 1 & 8,00 & 0 & 3 & 33 & 64 \\
Ilha - 1 & 11,00 & 0 & 46 & 10 & 44 \\
Ilha - 2 & 2,00 & 12 & 17 & 11 & 60 \\
Ilha - 2 & 8,00 & 0 & 1 & 26 & 73 \\
\hline
\end{tabular}

\section{Caracterização Física}

Os ensaios de Caracterização Física dos solos foram realizados seguindo as Normas da Associação Brasileira de Normas Técnicas. As amostras foram obtidas a partir de amostradores do tipo Shelby. Nos 0,10 m iniciais de cada amostra foram realizados ensaios de Limites de Atterberg com e sem secagem prévia seguindo a ABNT NBR 6459/2016 Versão Corrigida/2017 para o limite de liquidez e ABNT NBR 7180/2016 para o limite de plasticidade complementando os que já foram realizados para o projeto.

\section{Avaliação da Compressibilidade}

A compressibilidade do solo foi avaliada utilizando ensaios de compressão unidimencional de acordo com a ABNT NBR 16853/2020 em prensas do tipo Bishop com relação de braço de alavanca de 10:1. Dois tipos de anéis foram utilizados nas células de adensamento, um anel convencional de aço inoxidável de paredes finas, com pontas biseladas, área de 6000 $\mathrm{mm} 2$, altura de $20 \mathrm{~mm}$ e relação diâmetro/altura de 4,37, as pedras porosas foram colocadas no topo e na base do anel, para drenagem vertical. O segundo anel também de aço inoxidável com diâmetro de $100 \mathrm{~mm}$, altura de 30 mm, com relação de 
diâmetro/altura 3,33, a pedra porosa foi fixada na parede do cilindro de aço com espessura de 8,00 mm para os ensaios com drenagem horizontal (radial externa). Os anéis utilizados atendem as recomendações de Lambe (1951).

Nos ensaios foram utilizados dois tipos de amostras, uma indeformada obtida diretamente do amostrador Shelby e outra amolgada completamente em laboratório, quebrando a sua estrutura e remoldando em corpo de prova nas mesmas condições de campo (umidade e peso específico úmido). Na amostra amolgada, foi realizada a reconstrução da curva de compressibilidade do campo por Schmertmann (1953) e a estimativa da curva por Ábaco proposta por Oliveira (2002). A qualidade das amostras foi analisada pelos critérios de Lunne, Robertson e Powell (1997), Hong e Onitsuka (1998), Oliveira (2002) e Coutinho (2007).

Nos ensaios foram utilizados dois tipos de incremento de tensão, um com $\Delta \sigma / \sigma=1$, iniciando em $10 \mathrm{kPa}$ e até 1280 $\mathrm{kPa}$ em estágio com duração de $24 \mathrm{~h}$. O segundo, iniciava-se o carregamento com incremento $\Delta \sigma / \sigma=1$ partindo de $10 \mathrm{kPa}$ até a tensão de $40 \mathrm{kPa}$ (tensão próxima a tensão de pré-adensamento), a seguir, o incremento de tensão passa a ser menor do que 1 $(\Delta \sigma / \sigma<1)$, aplicando as tensões de $50,60,70$ e $80 \mathrm{kPa}$ e após a esta tensão, o incremento para ser igual a $1(\Delta \sigma / \sigma=1)$ até $1280 \mathrm{kP}$; todos os estágios de tensão tinham duração de 24h. Esta metodologia permite obter melhor a tensão de préadensamento (FERREIRA, 1982). No descarregamento a sequência foi 640, 160, 40 e $10 \mathrm{kPa}$.

\section{Avaliação da Resistência ao Cisalhamento}

A resistência não drenada foi avaliada em laboratório por ensaio triaxial consolidado não drenado (UU) e em campo por meio do ensaio "vane" e do piezocone de forma indireta. No ensaio triaxial, foram utilizados corpos de prova cilíndricos com diâmetro de $36 \mathrm{~mm}$ e altura de $100 \mathrm{~mm}$ moldados a partir de amostras indeformadas tipo Shelby. A velocidade de cisalhamento foi $0,77 \mathrm{~mm} / \mathrm{min}$, conforme recomendações de Lambe (1951) e aplicadas pressões confinantes de 50; 100; 200 e $300 \mathrm{kPa}$. A força vertical aplicada e a deformação foram medidas através de anel dinamométrico com capacidade de $100 \mathrm{kgf}$ e extensômetro mecânico com sensibilidade de $0,01 \mathrm{~mm}$, respectivamente.

No ensaio de "vane", utilizou-se palheta de $65 \mathrm{~mm}$ de diâmetro e $130 \mathrm{~mm}$ de altura que foi cravada estaticamente. A rotação da palheta foi feita por meio de motor elétrico, com capacidade de até $100 \mathrm{Nm}$ com velocidade de $0,1 \%$ seg. A aquisição dos dados foi realizada por meio de software específico, que fornece em tempo real a curva torque x rotação. O torque máximo permite obter resistência não drenada indeformada e após dez revoluções da palheta era medido o torque residual, que permite determinar a resistência não drenada amolgada para obter a sensibilidade do solo. Na Ilha 1 as determinações foram realizadas na profundidade $1,40 \mathrm{~m}$ e depois a cada metro de 2 a $12 \mathrm{~m}$, onde atingiu uma camada de areia no final. Na ilha 2 as determinações foram realizadas a 1,60;2,20;3; 6; 7; 8 e $9 \mathrm{~m}$ de profundidade quando a camada de areia foi atingida.

O ensaio penetração de cone com medida da poropressão (CPTU) seguiu as recomendações da ABNT, NBR 12069/(1991). O cone de aço com $1000 \mathrm{~mm} 2$ de área de ponta foi gravado de forma estática com velocidade constante de 20 $\mathrm{mm} / \mathrm{s}$ e leituras simultâneas de resistência de ponta (qc), resistência ao atrito lateral (fs), sobrepressão (U2), inclinação do cone e velocidade de cravação também eram realizadas. A cravação da ponteira do CPTU tinha capacidade de até 20 tf. Foram realizadas quatro dissipações em cada ilha. As pedras porosas foram saturadas antes do ensaio, em câmara de vácuo, para garantir a total desaeração do elemento. 


\section{Resultados e Discussão}

\section{Caracterização Física}

Os resultados dos Limites de Atterberg obtidos com a secagem prévia por Cadete (2016) e sem a secagem prévia por Rocha (2016) são apresentados na Tabela 2 para os solos da Ilha 1 e Ilha 2. Há uma variação de 3 a $28 \%$ nos valores do Limite de Liquidez e de 2 a 18 \% nos valores do Limite de Plasticidade. Os valores da variação no Limite de Liquidez com e sem secagem prévia do solo são inferiores aos encontrados na argila orgânica mole do bairro da Madalema quando calculados com os dados de secagem prévia de Ferreira, Amorim Jr \& Coutinho (1986) com os dados dos Limites de Liquidez sem secagem encontrados por Teixeira (1972) no solo do mesmo local que variou de 12,2 a 93,20 \%. Este procedimento mostra a importância da secagem prévia na determinação dos limites em solos orgânicos.

Tabela 2 - Valores dos Limites de Liquidez e Plasticidade com e sem secagem prévia (Rocha, 2016).

\begin{tabular}{|c|c|c|c|c|c|c|c|c|c|c|}
\hline \multirow{2}{*}{$\begin{array}{l}\text { Secagem } \\
\text { prévia }\end{array}$} & \multicolumn{2}{|c|}{ Ilha 1} & \multicolumn{2}{|c|}{ Ilha 2} & \multicolumn{2}{|c|}{ Ilha 1} & \multicolumn{2}{|c|}{ Ilha 2} & \multicolumn{2}{|c|}{ Ilha 1} \\
\hline & Sem & Com & Sem & Com & Sem & Com & Sem & Com & Sem & Com \\
\hline Prof. (m) & \multicolumn{4}{|c|}{----------------2,00---------------- } & \multicolumn{4}{|c|}{----------------8,00---------------- } & \multicolumn{2}{|c|}{-----11,00----- } \\
\hline $\mathrm{WL}(\%)$ & 49,70 & 38,00 & 50,90 & 55,00 & 95,00 & 68,00 & 78,80 & 65,00 & 27,70 & 25,00 \\
\hline $\mathrm{WP}(\%)$ & 36,90 & 26,00 & 41,29 & 32,00 & 57,88 & 41,00 & 49,61 & 37,00 & 17,49 & 16,00 \\
\hline IP $(\%)$ & 12,80 & 12,00 & 9,61 & 23,00 & 37,12 & 27,00 & 29,19 & 28,00 & 10,21 & 9,00 \\
\hline
\end{tabular}

Fonte: Autores.

\section{Compressibilidade da Argila}

As curvas de variação do índice de vazios versus tensão aplicada de consolidação $(\sigma \mathrm{vc})$, em log, são apresentadas na Figura 3 para ensaios de compressão unidimensional com drenagem vertical e radial. As curvas de compressibilidade para o solo da Ilha 1 (Figura 3a) e da Ilha 2 (Figura 3b) são bastantes semelhantes. Na profundidade de $2 \mathrm{~m}$ a curva de compressibilidade do solo Ilha 1 apresenta pequena diferença na forma, considerada uma exceção às demais curvas, isto é devido a própria heterogeneidade da amostra e nesta profundidade apresenta um OCR de 3,99 que pode ser devido a algum ressecamento. Nos solos da Ilha 2 são quase superpostas. As curvas apresentam dois trechos retilíneos na compressão virgem, o primeiro de 80 a $320 \mathrm{kPa}$ e o segundo de 320 a $1280 \mathrm{kPa}$, comportamento similar foi encontrado por Coutinho (1976), Ferreira (1982), Oliveira (2002) e Bello et al (2019). A declividade do segundo trecho é menor do que o primeiro. Segundo Ferreira (1982) esta característica é encontrada tipicamente nas argilas normalmente adensadas, estruturadas e em amostras de boa qualidade.

As Tabelas 3 e 4 apresentam os valores do índice de vazios inicial, tensão de pré-adensamento e índices de compressão e expansão dos solos da Ilha 1 e da Ilha 2, respectivamente. Nos solos da Ilha 1 , o índice de vazios nas profundidades de $2 \mathrm{~m}$ e $8 \mathrm{~m}$ é, em média, 3, 4 vezes superior ao da profundidade de $11 \mathrm{~m}$, em consequência é menos compressível. Nos solos da Ilha 2, o índice de vazios na profundidade de $8 \mathrm{~m}$ é superior ao da profundidade de $2 \mathrm{~m}$, em média, 1, 3 vezes, sendo mais compressível. Praticamente não há diferença nos valores da tensão de pré-adensamento obtidas pelos métodos de Pacheco Silva e Casagrande para os solos das duas Ilhas. A tensão de pré-adensamento é melhor definida quando se aplicam incrementos de tensão $\Delta \sigma / \sigma<1$ principalmente quando se determina pelo método de Casagrande. 
Figura 3 - Curvas de variação do Índice de vazios com a tensão de consolidação aplicada: a) solo da Ilha 1, b) solo da Ilha 2.

a)

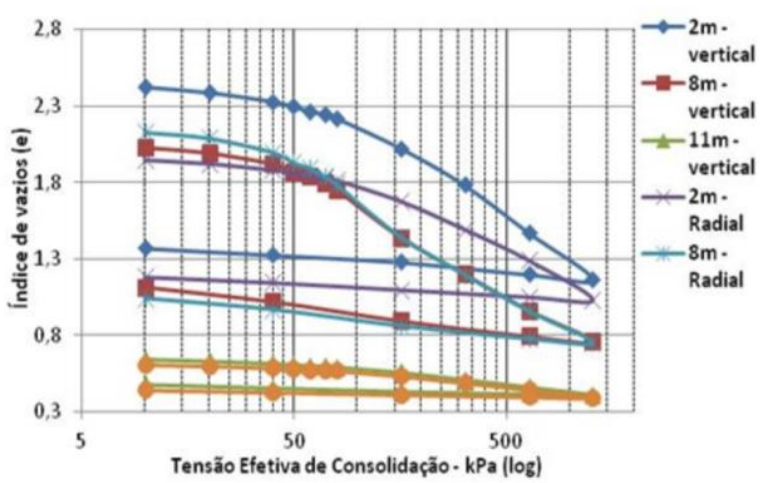

b)

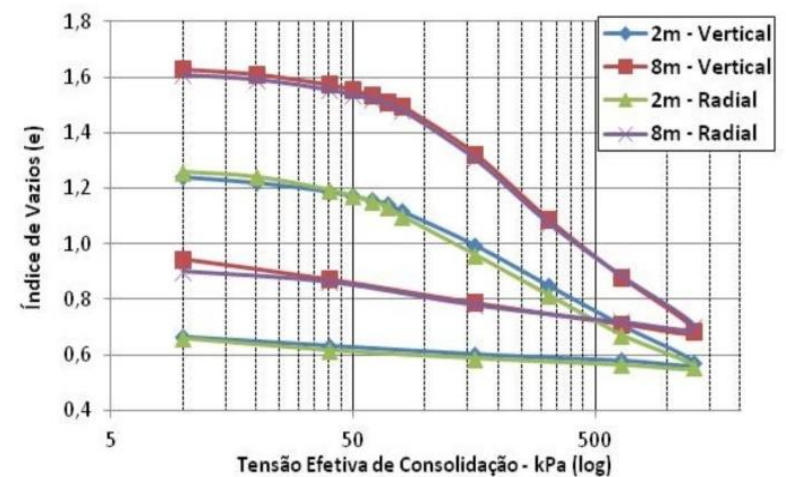

Fonte: Autores.

Tabela 3 - Valores do índice de vazios inicial, tensão de pré-adensamento e índices de compressão e expansão dos solos da Ilha 1.

\begin{tabular}{|c|c|c|c|c|c|c|c|c|}
\hline \multicolumn{9}{|c|}{ Ilha 1} \\
\hline \multirow{3}{*}{$\begin{array}{c}\text { Prof. (m) } \\
\text { Ensaio }\end{array}$} & \multicolumn{2}{|c|}{------ 2,00 ------ } & \multicolumn{4}{|c|}{------------------ 8,00 ------------------ } & \multicolumn{2}{|c|}{----- 11,00 ------ } \\
\hline & \multirow[t]{3}{*}{ Vertical } & \multirow{3}{*}{ Radial } & \multicolumn{2}{|c|}{ Vertical } & \multicolumn{2}{|c|}{ Radial } & \multirow[t]{3}{*}{ Vertical } & \multirow[t]{3}{*}{ Radia } \\
\hline & & & $1^{\circ}$ trecho & $2^{\circ}$ trecho & $1^{\circ}$ trecho & $2^{\circ}$ trecho & & \\
\hline Trecho Reto & & & $\begin{array}{c}80-320 \\
(\mathrm{kPa})\end{array}$ & $\begin{array}{c}320-1280 \\
(\mathrm{kPa})\end{array}$ & $\begin{array}{c}80-320 \\
(\mathrm{kPa})\end{array}$ & $\begin{array}{c}320-1280 \\
\mathrm{kPa}\end{array}$ & & \\
\hline $\mathrm{e}_{0}$ & 2,494 & 1,981 & \multicolumn{2}{|c|}{2,074} & \multicolumn{2}{|c|}{2,168} & 0,666 & 0,623 \\
\hline $\begin{array}{c}\sigma_{\mathrm{vm}}(\mathrm{kPa}) \\
\text { Pacheco Silva }\end{array}$ & 90,00 & 100,00 & \multicolumn{2}{|c|}{45,00} & \multicolumn{2}{|c|}{40,00} & 67,00 & 75,00 \\
\hline $\begin{array}{c}\sigma_{\mathrm{vm}}(\mathrm{kPa}) \\
\text { Casagrande }\end{array}$ & 95,00 & 100,00 & \multicolumn{2}{|c|}{54,00} & \multicolumn{2}{|c|}{48,00} & 80,00 & 83,00 \\
\hline $\mathrm{Cc}$ & 0,914 & 0,642 & 0,915 & 0,728 & 0,978 & 0,740 & 0,159 & 0,150 \\
\hline Cs & 0,105 & 0,070 & \multicolumn{2}{|c|}{0,188} & \multicolumn{2}{|c|}{0,151} & 0,035 & 0,026 \\
\hline
\end{tabular}

$\mathrm{e}_{0}$ - Índice de vazios inicial; ovm - tensão de pré-adensamento; Cc - índice de compressão; Cs - Índice de expansão. Fonte: Autores.

Tabela 4 - Valores do índice de vazios inicial, tensão de pré-adensamento e índices de compressão e expansão dos solos da Ilha 2.

\begin{tabular}{|c|c|c|c|c|c|c|}
\hline Prof. (m) & \multicolumn{2}{|c|}{------ 2,00 ------ } & \multicolumn{4}{|c|}{------------------- 8,00 ------------------- } \\
\hline Ensaio & Vertical & Radial & \multicolumn{2}{|c|}{ Vertical } & \multicolumn{2}{|c|}{ Radial } \\
\hline & & & $1^{\circ}$ trecho & $2^{\circ}$ trecho & $1^{\circ}$ trecho & $2^{\circ}$ trecho \\
\hline Trecho Reto & & & $\begin{array}{c}80-320 \\
(\mathrm{kPa})\end{array}$ & $\begin{array}{c}320-1280 \\
(\mathrm{kPa})\end{array}$ & $\begin{array}{c}80-320 \\
(\mathrm{kPa})\end{array}$ & $\begin{array}{c}320-1280 \\
(\mathrm{kPa})\end{array}$ \\
\hline $\mathrm{e}_{0}$ & 1,277 & 1,281 & \multicolumn{2}{|c|}{1,670} & \multicolumn{2}{|c|}{1,637} \\
\hline$\sigma_{\mathrm{vm}}(\mathrm{kPa})$ Pacheco Silva & 60,00 & 44,00 & \multicolumn{2}{|c|}{66,00} & \multicolumn{2}{|c|}{66,00} \\
\hline$\sigma_{\mathrm{vm}}(\mathrm{kPa})$ Casagrande & 67,00 & 53,00 & \multicolumn{2}{|c|}{76,00} & \multicolumn{2}{|c|}{69,00} \\
\hline $\mathrm{Cc}$ & 0,478 & 0,677 & 0,676 & 0,649 & 0,677 & 0,618 \\
\hline Cs & 0,048 & 0,060 & \multicolumn{2}{|c|}{0,129} & \multicolumn{2}{|c|}{0,102} \\
\hline
\end{tabular}

$\mathrm{e}_{0}$ - Índice de vazios inicial; $\sigma_{\mathrm{vm}}$ - tensão de pré-adensamento; Cc - índice de compressão; Cs - Índice de expansão Fonte: Autores. 
A estimativa da velocidade com que o excesso de pressão nos poros é dissipada requer a determinação do coeficiente de adensamento que traduza adequadamente os efeitos da solicitação e das condições de contorno (drenagem). A variação dos coeficientes de adensamento com drenagem vertical $(\mathrm{Cv})$ e horizontal $(\mathrm{Ch})$ com a tensão vertical média aplicada é apresentada na Figura 4 para os solos das duas ilhas de investigação. Os valores do $\mathrm{Cv}$ e Ch decresceram com o acréscimo da tensão aplicada, sendo maior no trecho de recompressão (antes a tensão de pré-adensamento) do que no trecho de compressão virgem (após a tensão de pré-adensamento). No trecho de compressão virgem a razão $\mathrm{Ch} / \mathrm{Cv}$ variou de 1,03 a 11,17 com média de 3,94. Os valores da razão $\mathrm{Ch} / \mathrm{Cv}$ no trecho de compressão virgem a 8,0 m de profundidade no solo da Ilha 1 variaram de 1,29 a 3,79 e no solo da Ilha 2 varia de 1,76 a 3,76. Ferreira (1982) obteve valores de razão $\mathrm{Ch} / \mathrm{Cv}$ variando de 1,00 a 3,50 na argila do bairro da Madalena -Recife/PE e Bello (2011) encontrou valores que variaram de 0,90 a 10,50 na argila de Suape/PE.

Figura 4 - Curvas do Coeficiente de Adensamento com a Tensão Vertical Média: a) Vertical e b) Horizontal.

a)

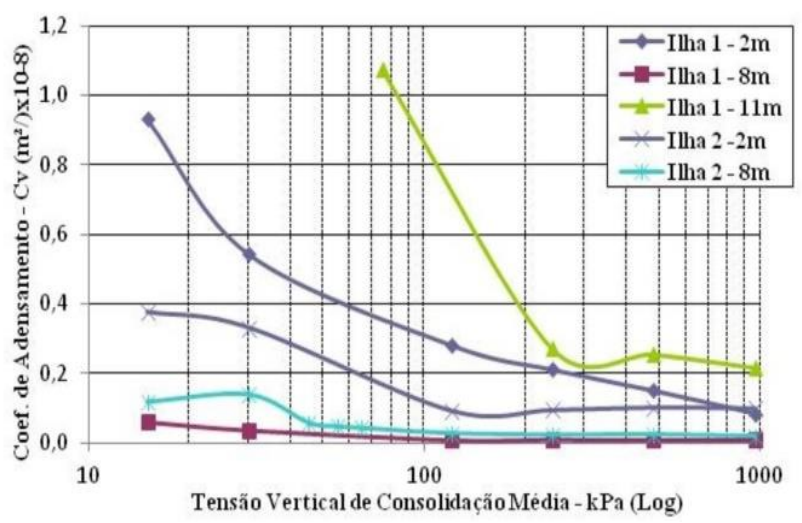

b)

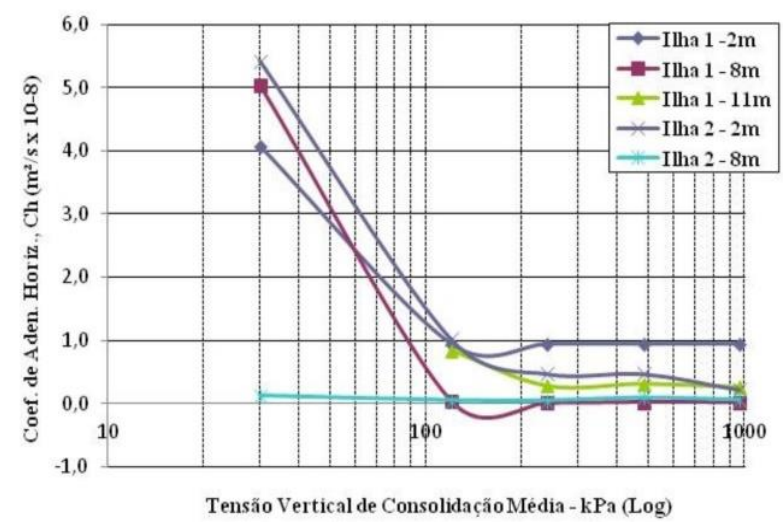

Fonte: Autores.

Os valores das tensões efetivas das terras, das tensões de pré-adensamento e os valores do OCR com a profundidade são apresentados na Tabela 5. A Figura 5a mostra as curvas de variação do índice de vazios versus tensão vertical de consolidação para a amostra de boa qualidade, para a amostra completamente amolgada em laboratório (remoldada na mesma condição de amostra de boa qualidade), a curva de reconstituição de campo proposta por Schmertmann (1953) e a curva estimada pela proposta de Oliveira (2002) que reconstitui a curva de compressibilidade baseada em valores de um ábaco contendo dados e parâmetros de amostras de boa qualidade coletadas pelo amostrador Sherbrooke. No trecho retilíneo compressão virgem as curvas de reconstituição proposta por Schmertmann (1953) e de Oliveira (2002) são praticamente paralelas, fato que não se verifica na amostra completamente amolgada em laboratório. A tensão de pré-adensamento determinada na amostra de boa qualidade é muito próxima da determinada na curva de reconstituição de campo proposta por Schmertmann (1953) e difere (inferior) na curva estimada pela proposta Oliveira (2002) e de forma superior a obtida na amostra completamente amolgada em laboratório. Ferreira (1982) e Oliveira (2002) mostram que o amolgamento causa uma redução na tensão de pré-adensamento e no coeficiente de adensamento vertical no trecho de recompressão. A história de tensão (OCR versus profundidade, Figura 5b) indica que o depósito é ligeiramente pré-adensado a normalmente adensado, com exceção da profundidade de 2 metros na Ilha 1 que a amostra pode ter sofrido algum ressecamento decorrente da oscilação do lençol freático ou pela própria heterogeneidade da amostra. A razão de pré-adensamento (OCR) diminuiu com a profundidade característica típica de depósitos de argilas moles do Recife como foi observado por Ferreira (1982) e Oliveira (1991) e Bello 
(2004).

Tabela 5 - Valores de Razão de Pré-adensamento (OCR) para os solos da Ilha 1 e Ilha 2.

\begin{tabular}{cccccccc}
\hline \multicolumn{3}{c}{ Ilha 1 } & \multicolumn{4}{c}{ Ilha 2 } \\
\hline $\begin{array}{c}\text { Prof. } \\
(\mathrm{m})\end{array}$ & $\begin{array}{c}\text { Tensão Efetiva } \\
\text { de Campo }(\mathrm{kPa})\end{array}$ & $\begin{array}{c}\text { Tensão de pré- } \\
\text { adensamento }(\mathrm{kPa})\end{array}$ & OCR & $\begin{array}{c}\text { Prof. } \\
(\mathrm{m})\end{array}$ & $\begin{array}{c}\text { Tensão Efetiva } \\
\text { de Campo }(\mathrm{kPa})\end{array}$ & $\begin{array}{c}\text { Tensão de pré- } \\
\text { adensamento }(\mathrm{kPa})\end{array}$ & OCR \\
\hline 2,0 & 23,80 & 95,00 & 3,99 & 2,00 & 33,90 & 67,00 & 1,98 \\
8,0 & 37,60 & 54,00 & 1,44 & 8,00 & 46,50 & 76,00 & 1,63 \\
11,0 & 58,40 & 80,00 & 1,37 & & & & \\
\hline
\end{tabular}

Fonte: Autores (2022).

Figura 5 - História de tensão: a) Curvas de Compressibilidade e correções por Schmertmann (1953) e por Oliveira (2002); b) Variação de OCR versus profundidade (m).

a)

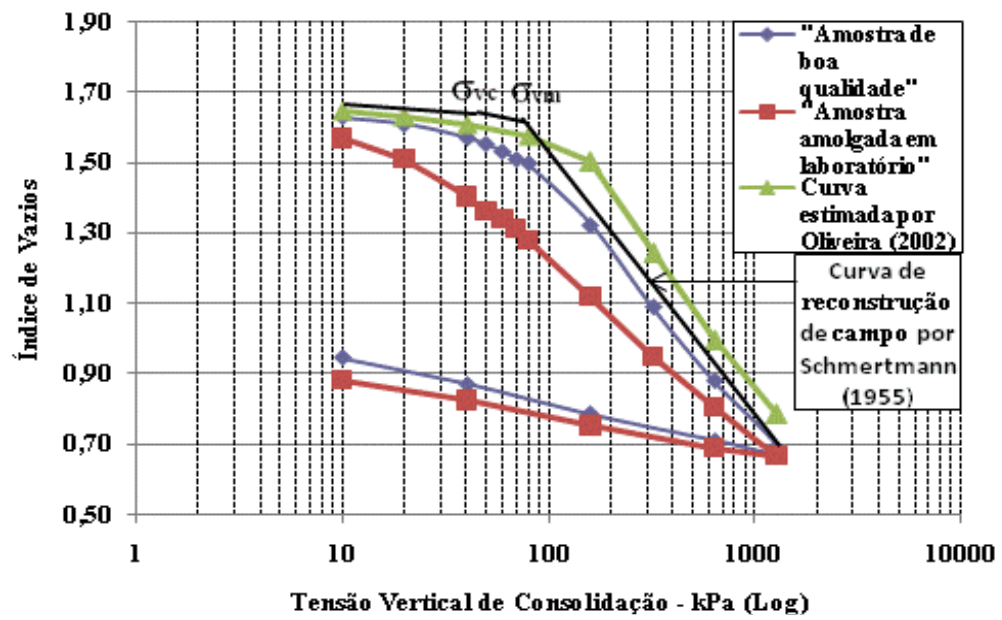

Fonte: Autores. b)

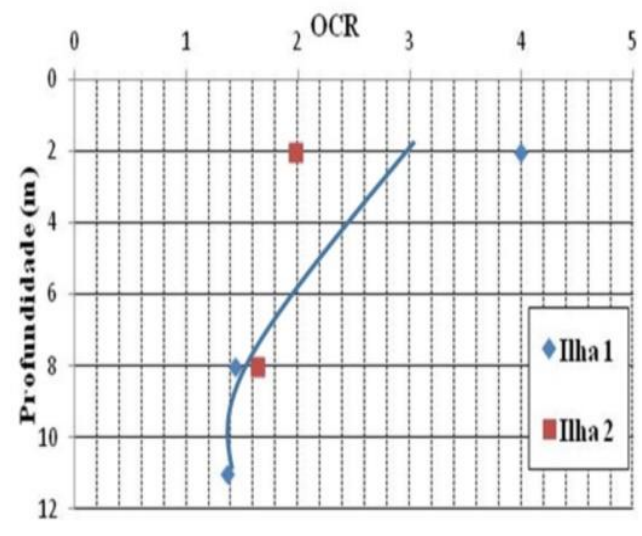

A qualidade das amostras foi analisada por quatro critérios. Os critérios de Lunne, Robertson e Powell (1997), Hong e Onitsuka (1998) são apresentados nas Tabelas 6 e 7. A Tabela 8 apresenta o critério de Oliveira (2002), que sugeriu uma Nota (N) para determinar a qualidade das amostras de forma direta e objetiva se utilizando como referência de cálculo o Grau de Amolgamento (SD\%) apresentado pelo critério de Hong e Onitsuka (1998) e a Tabela 9 apresenta o critério de Coutinho (2007). Pelos critérios de Lunne, Robertson \& Powell (1997), Hong \& Onitsuka (1998) e Coutinho (2007) as amostras são classificadas como boa a regular. A exceção ficou por conta da amostra da Ilha 1 aos 11 metros que é classificada como pobre por Lunne, Robertson \& Powell (1997) e Coutinho (2007), porém o critério deste último autor limita o OCR a 2,5, fato que não encontra aplicação na classificação da amostra da Ilha 1 aos 2 metros de profundidade, que apresentou OCR em torno de 4.

Tabela 6 - Classificação por Lunne, Robertson e Powell (1997)

\begin{tabular}{|c|c|c|c|c|c|c|}
\hline \multirow{3}{*}{ Prof. (m) } & \multicolumn{3}{|c|}{ Classificação - Ilha 1} & \multicolumn{3}{|c|}{ Classificação - Ilha 2} \\
\hline & \multicolumn{3}{|c|}{ Critério Lunne } & \multicolumn{3}{|c|}{ Critério Lunne } \\
\hline & OCR & $\Delta \mathrm{e} / \mathrm{e}$ & Classificação & OCR & $\Delta \mathrm{e} / \mathrm{e}$ & Classificação \\
\hline 2,00 & 3,99 & 0,051 & Regular & 1,98 & 0,072 & Regular \\
\hline 8,00 & 1,44 & 0,071 & Regular & 1,64 & 0,048 & Regular \\
\hline 11,00 & 1,37 & 0,093 & Pobre & - & - & - \\
\hline
\end{tabular}


Tabela 7 - Classificação por Hong e Onitsuka (1998).

\begin{tabular}{ccccccccc}
\hline & \multicolumn{4}{c}{ Classificação - Ilha 1 } & \multicolumn{4}{c}{ Classificação - Ilha 2 } \\
\cline { 2 - 9 } Prof. (m) & \multicolumn{4}{c}{ Critério Hong e Onitsuka } & \multicolumn{4}{c}{ Critério Hong e Onitsuka } \\
& WL & CLR & CLB & SD\% & WL & CLR & CLB & SD\% \\
\hline 2,00 & 1,696 & 0,17 & 0,047 & 27,14 & 1,707 & 0,18 & 0,039 & 22,08 \\
8,00 & 1,978 & 0,27 & 0,059 & 22,13 & 1,897 & 0,24 & 0,035 & 14,60 \\
11,00 & 1,442 & 0,09 & 0,029 & 32,62 & - & - & - \\
\hline
\end{tabular}

CLB - Inclinação do trecho de recompressão da amostra natural, CLR - Inclinação do trecho de recompressão para amostra completamente amolgada, SD - Grau de amolgamento.. Fonte: Autores.

Tabela 8 - Classificação por Oliveira (2002).

\begin{tabular}{ccccc}
\hline & \multicolumn{2}{c}{ Classificação - Ilha 1 } & \multicolumn{2}{c}{ Classificação - Ilha 2 } \\
\cline { 2 - 5 } Prof. $(\mathrm{m})$ & \multicolumn{4}{c}{ Nota proposta por Oliveira $(2002)$} \\
& $(\mathrm{N})=(100-\mathrm{SD} \%) / 10$ & Classificação & $(\mathrm{N})=(100-\mathrm{SD} \%) / 10$ & Classificação \\
\hline 2,00 & 7,29 & Boa & 7,79 & Boa \\
8,00 & 7,79 & Boa & 8,54 & Boa \\
11,00 & 6,74 & Regular & - & - \\
\hline
\end{tabular}

$\mathrm{N}$ - nota. Fonte: Autores.

Tabela 9 - Classificação por Coutinho (2007).

\begin{tabular}{|c|c|c|c|c|c|c|}
\hline \multirow{3}{*}{ Prof. (m) } & \multicolumn{4}{|c|}{ Classificação - Ilha 1} & \multicolumn{2}{|c|}{ Classificação - Ilha 2} \\
\hline & \multicolumn{6}{|c|}{ Critério Coutinho (2007) } \\
\hline & OCR & $\Delta \mathrm{e} / \mathrm{e}$ & Classificação & OCR & $\Delta \mathrm{e} / \mathrm{e}$ & Classificação \\
\hline 2,00 & 3,99 & 0,051 & Boa a Regular & 1,98 & 0,072 & Boa a Regular \\
\hline 8,00 & 1,44 & 0,071 & Boa a Regular & 1,64 & 0,048 & Muito Boa a Excelente \\
\hline 11,00 & 1,37 & 0,093 & Pobre & - & - & - \\
\hline
\end{tabular}

OCR - Razão de Pré-adensamento, $\Delta \mathrm{e}$ - variação do índice de vazios, e - índice de vazios. Fonte: Autores.

\section{Resistência Não Drenada}

A Figura 6 apresenta os resultados da resistência não drenada das Ilhas 1 (Figura 6a) e Ilha 2 (Figura 6b), para os ensaios Triaxial (UU), Vane Test natural e amolgado, e Piezocone. A comparação dos resultados dos ensaios de Piezocone, Vane e Triaxial UU mostra que na Ilha 1 há uma maior concordância entre os dois últimos nas camadas 2 e $8 \mathrm{~m}$, mas aos $11 \mathrm{~m}$ os valores do Piezocone e Triaxial UU são iguais e o do Vane é um pouco superior. Na Ilha 2, os valores da resistência obtidos pelos três ensaios são próximos em todas as camadas avaliadas. Observa-se que o depósito de solo mole apresenta duas subcamadas: uma até os $4 \mathrm{~m}$ e a outra dos 4 aos $12 \mathrm{~m}$ de profundidade. Nos quatro metros iniciais, a resistência não drenada é em média $35 \mathrm{kPa}$ e, nos oito metros seguintes, fica em torno de $25 \mathrm{kPa}$.

O valor da resistência não drenada é estimado pelo piezocone utilizando parâmetros experimentais: Nkt - fator de capacidade de carga, Nke - fator de poro-pressão e $\mathrm{N} \Delta \mathrm{t}$ - fator de resistência de ponta efetiva. Neste trabalho, o valor adotado para estimar a resistência não drenada foi $\mathrm{Nkt}=14$.

Nas argilas moles de Suape, Bello (2011) encontrou valores de Nkt situados entre 10,0 e 15,5. Oliveira (2000) considerou adequado o valor de Nkt igual a 11 para a primeira camada dos depósitos argilosos da Madalena e do SESI-Ibura, também no Recife. No entanto, o autor determinou o valor de Nkt a partir de valores de Su (resistência não drenada) obtidos através de ensaios de palheta de campo e foram encontrados valores médios situados entre 11,3 e 13,1. Para discutir o valor adotado neste trabalho em comparação com outros autores, foi encontrado através da Equação 1 um valor de Nkt para cada valor de resistência não drenada Su obtido pelo ensaio de palheta de campo nas ilhas de investigação deste trabalho. 
Figura 6 - Resistência não drenada: a) Ilha 1, b) Ilha 2, c) Sensibilidade do depósito na Ilha 1 (VT 01) e Ilha 2 (VT 02 ).

a)

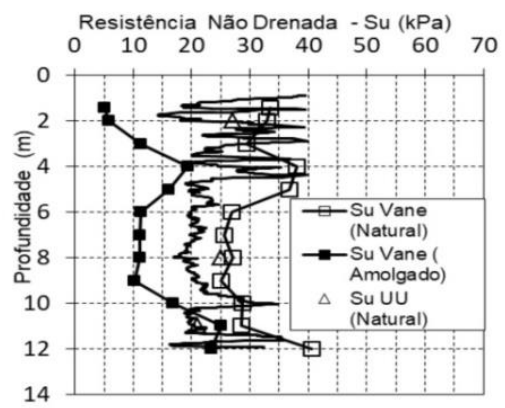

$\mathrm{Nkt}=\left(\mathrm{qt}-\sigma \mathrm{v}_{0}\right) / \mathrm{Su}$ b)

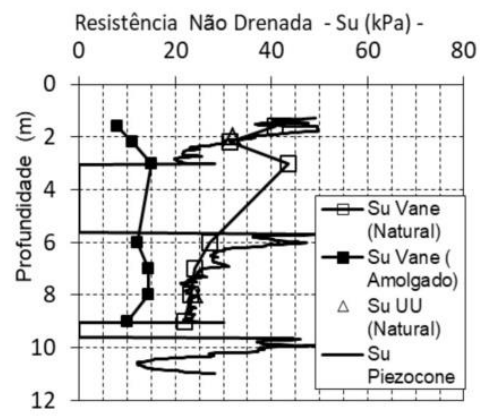

c)

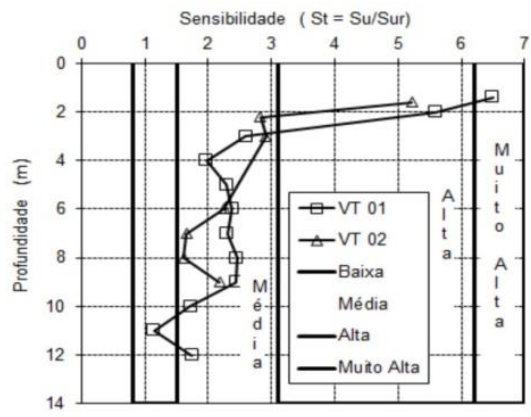

(1)

Fonte: Autores.

No solo da Ilha 1, o valor médio encontrado foi Nkt igual a 10 e na Ilha 2 um valor de Nkt médio igual a 14. Assim, é adequado sugerir que para as argilas moles analisadas sejam utilizados valores médios de Nkt iguais a $12 \pm 2$, tendo como referência o ensaio de palheta de campo.

Para determinar a sensibilidade (St) e a importância de sua estrutura, foi calculada a razão entre a resistência não drenada do solo no estado indeformado e a resistência do solo no estado amolgado. A avaliação da sensibilidade (Figura 7c) mostrou que a argila é mais sensível nos quatro metros iniciais nas duas ilhas de investigação. O solo da Ilha 1 apresentou sensibilidade média de 2,76, e da ilha 2 uma média de 2,66. Porém, nos quatro metros iniciais, a sensibilidade chega a 6,50 e 5,22 nas ilhas 1 e 2, respectivamente. Esses valores se aproximam daqueles encontrados por Oliveira (2000) para as primeiras camadas da argila do bairro da Madalena (média de 6,4) e no SESI-Ibura (média de 6,1). Segundo a classificação de Skempton \& Northey (1952), a argila nos 4,0 m iniciais é sensível (St entre 4 e 8) e para profundidades maiores a sensibilidade é média (St entre 2 e 4). Rocha et al. (2016) estudou a história de tensão do depósito realizando ensaios de adensamento com acréscimo de tensão menor do que 1 próximo da tensão de pré-adensamento. A Tabela 10 mostra os valores da tensão de préadensamento, da razão de pré-adensamento (OCR). Esses dados foram utilizados para obter a resistência não drenada através da correlação $\mathrm{Su}=0,22 \sigma \mathrm{vm}$ porposta por Mesri (1975).

Tabela 10 - Valores da tensão $\left(\sigma_{\mathrm{vm}}\right)$ e razão de pré-adensamento (OCR) e estimativa da resistência não drenada (Su) pela proposta de Mesri (1975).

\begin{tabular}{ccccc}
\hline Local & $\begin{array}{c}\text { Prof. } \\
(\mathrm{m})\end{array}$ & $\begin{array}{c}\sigma_{\mathrm{vm}} \\
(\mathrm{kPa})\end{array}$ & OCR & $\begin{array}{c}\mathrm{Su}=0,22 \sigma_{\mathrm{vm}} \\
(\mathrm{kPa})\end{array}$ \\
\hline \multirow{2}{*}{ Ilha 1 } & 2,00 & 95,00 & 3,99 & 20,90 \\
& 8,00 & 54,00 & 1,44 & 11,88 \\
& 11,00 & 80,00 & 1,37 & 17,60 \\
Ilha 2 & 2,00 & 67,00 & 1,98 & 14,74 \\
& 8,00 & 76,00 & 1,64 & 16,72 \\
\hline
\end{tabular}

Fonte: Autores. 
$\mathrm{O}$ depósito tem um maior pré-adensamento nas profundidades iniciais $(\mathrm{OCR}=3,99) \mathrm{e}$, à medida que a profundidade cresce, é levemente pré-adensado $(\mathrm{OCR}=1,34)$. Isto se deve ao processo de formação do depósito com regressões e transgressões, ressecamento das camadas mais superficiais, variação do nível d'água além da compressão secundária (Ferreira, 1982). Os valores da resistência (Su) estimados pela proposta de Mesri (1975) para os solos das duas ilhas são próximos aos valores obtidos nos ensaios de CPTU e inferiores aos valores obtidos nos ensaios UU e Vane. Esses valores são conservadores.

\section{Conclusões}

Com o objetivo principal de analisar o comportamento tensão-deformação-resistência de uma argila orgânica mole da zona Norte da cidade do Recife-PE, destacam-se as principais conclusões: o subsolo é constituído de uma camada superficial de areia siltosa e silte arenoso seguido de camadas de aluviões muito moles com presença de turfa e argila de baixa resistência; a secagem prévia da argila orgânica influencia na determinação dos limites de liquidez e plasticidade; é importante avaliar a qualidade da amostra em argila orgânica mole na determinação dos parâmetros hidrogeomecânicos do solo e mesmo em amostras de boa qualidade é necessário fazer correções das curvas de compressibilidade para melhor definir tensão de préadensamento, as investigações de campo e de laboratório na argila orgânica mole na região Norte da cidade contribuem com o avanço do conhecimento das argilas moles da Planície do Recife-PE.

A argila é levemente pré-adensada até 4,0 m, normalmente adensada a maiores profundidades e a relação entre os coeficientes de adensamento radial e vertical $(\mathrm{Ch} / \mathrm{Cv})$ é em média de 3,94. A resistência não drenada média nos quatro metros iniciais (35 kPa) é maior do que em profundidades superiores $(25 \mathrm{kPa})$, devido ao efeito do pré-adensamento apresentando também maior sensibilidade. Os valores do Fator de Capacidade de Carga (Nkt) obtidos por retonálise variaram de $12 \pm 2$, indicando um valor médio um pouco inferior ao normalmente utilizado $(\mathrm{Nkt}=14)$ nos ensaios de piezone nas argilas moles do Recife.

Como sugestões para novas pesquisa é indicado avaliar a influência da secagem prévia das amostras de argilas orgânicas na caracterização física do solo e ampliar os estudos do comportamento tensão-deformação-resistência em novos locais de depósitos de argilas orgânicas mole.

\section{Agradecimentos}

Ao Consórcio Projetec/ABF/Norconsult pela disponibilização dos dados necessários para o desenvolvimento deste trabalho.

\section{Referências}

Almeida, M. S. S.; Futai, M. M.; Lacerda, W. A., Marques, M. E. S. (2008). Laboratory behaviour of Rio de Janeiro soft clays. Part 1: index and compression properties. Soils \& Rocks, 31(2), 69-75.

Amorim Jr. W. M. (1975). Contribuição ao estudo das argilas orgânicas de Pernambuco. Dissertação Mestrado em Engenharia Civil, COPPE/ UFRJ, Rio de Janeiro. 101p. http://www.coc.ufrj.br/pt/dissertacoes-de-mestrado/75-1975/318-washington-moura-de-amorim-junior

Associação Brasileira de Normas Técnicas - ABNT NBR 12069. (1991) Solo - Ensaio de penetração de cone in situ (CPT). Rio de Janeiro.

Associação Brasileira de Normas Técnicas. (ABNT) NBR 6459. (2017). Versão Corrigida: Solo - Determinação do Limite de Liquidez. Rio de Janeiro.

Associação Brasileira De Normas Técnicas. (ABNT) NBR 7180. (2016). Solo - Determinação de Limite de Plasticidade. Rio de Janeiro.

Associação Brasileira de Normas Técnicas. (ABNT) NBR 16853. (2020): Solo - Ensaio de Adensamento Unidimensional. Rio de Janeiro. 
Bello, M. I. M. 2004. Estudo de ruptura em aterros sobre solos moles: aterro do galpão localizado na BR 101 - PE. Dissertação Mestrado em Engenharia Civil, UFPE, Recife. Pernambuco, Brasil. 231p. https://repositorio.ufpe.br/handle/123456789/5692.

Bello, M. I. M. 2011. Parâmetros geotécnicos e banco de dados de argilas moles: o caso de Suape. Tese de Doutorado em Engenharia Civil UFPE, Recife, Pernambuco, Brasil. 319p.

Bello, M. I., Marques, C. V., Coutinho, R. Q., \& Noberto, A. S. (2019). Evaluation of sample quality and correction of compressibility and strength parameters experience with Brazil soft soils. Soils \& Rocks. São Paulo 42(3), 245-263. http://www.soilsandrocks.com.br/soils-androcks/SR42-3_245-263.pdf

Bressani, L. A., Núñez W.P. Schmitt, L. A., Rizzon, M. M. Victorino, A. R. Hirsch, F., Brito L., Vitorello, T. (2011). Caracterização de depósitos de argilas moles na região metropolitana de Porto Alegre e estudo preliminar de melhorias desses solos por meio da adição de cal Revista Antt, . 3(2), 1-10.

Cadete, A. N. M. (2016). Avaliação da resistência não drenada de uma argila orgânica mole do bairro de Chão de Estrelas em Recife-PE. Dissertação de Mestrado em Engenharia Civil, UFPE, Recife, Pernambuco, Brazil. 164p.

https://repositorio.ufpe.br/bitstream/123456789/22324/4/DISSERTA\%C3\%87\%C3\%83O\%20Ana\%20Nery\%20de\%20Macedo\%20Cadete.pdf .

Carvalho, J. B. A. (1975). Algumas propriedades físico-químicas e de engenharia de uma argila orgânica mole do Recife. Dissertação Mestrado em Engenharia Civil, UFPB, Campina Grande, Paraíba, Brasil.152p. http://dspace.sti.ufcg.edu.br:8080/jspui/handle/riufcg/1857.

Coêlho, L. B. M. (1997). Considerações a respeito de um ensaio alternativo para determinação do coeficiente de adensamento horizontal de solos. Dissertação de Mestrado, COPPE/UFRJ, Rio de Janeiro.164p. http://www.coc.ufrj.br/pt/dissertacoes-de-mestrado/97-1997/1414-luciana-bezerra-de-mellocoelho.

Costa, A. J. (1960). O subsolo do Recife. Separata do Boletim Técnico da Secretaria de Viação e Obras / ITEP. Recife, Pernambuco, Brasil.

Coutinho, R. Q. (1976). Características de adensamento com drenagem vertical e radial em argila mole da Baixada Fluminense. Dissertação de Mestrado em Engenharia Civil, COPPE/UFRJ, Rio de Janeiro. 213p. https://pantheon.ufrj.br/browse?type=author\&value=Coutinho\%2C+Roberto+Quental.

Coutinho, R. Q. (1980). Estudo da caracterização geotécnica da argila orgânica do Recife. Pesquisa CNPq, Relatório de Atividades.Recife, Pernambuco, Brasil.

Coutinho, R. Q. (2007) Characterization and Engineering Properties of Recife Soft Clays - Brazil. In: Tan, Phoon, Higth and Leroueil (editors). Characterization and Engineering Properties of Natural Soils. In: Proceeding of the Second International Workshop on Characterization e Engineering Properties of Natural Soils. Taylor \& Francis/Balkema, 3, 2049-2100.

Coutinho, R. Q., Oliveira, J. T. R. \& Danziger, F. A. B. (1993). Caracterização geotécnica de uma argila mole do Recife. Solos e Rochas, 16( 4), 255-266.

Ferreira, S. R. M. (1982). Compressibilidade de uma argila mole do Recife. Dissertação de Mestrado em Engenharia Civil, COPPE/UFRJ, Rio de Janeiro. 212p. https://pantheon.ufrj.br/bitstream/11422/3268/1/156938.pdf.

Ferreira, S. R. M., Amorim Jr, W. N \& Coutinho, R. Q. (1986). Argila orgânica do Recife - contribuição ao banco de dados. In: VIII Cong. Bras. Mec. Solos e Eng. de Fund., ABMS, 1. 183 - 197. Porto Alegre, Brasil.

Hong, Z., Onitsuka, K. (1998). Method of Correcting Yield Stress and Compression Index of Ariake Clays for Sample Disturbance. Soils and Foundations, 38(2). $211-222$.

Lambe, T. W. (1951). Soil Testing for engineers. New York: John Wiley.

Lunne, T., Robertson, P. K. \& Powell, J. J. M. (1997). Cone penetration Testing in Geotechnical practice. Blackie Academic \& Professional.

Magalhães, D. F. V., Bello, M. I. M. C. V.\& Norberto, A. S. (2020). Dimensionamento de geossintético para reforço de aterro sobre solo mole. Research, Society and Development, 9 (8), p. e355985323, http://dx.doi.org/10.33448/rsd-v9i8.5323.

Mesri, G. (1975). New Design Procedure for Stability of Soft Clays. Journal of the Geotechnical Engineering Division, ASCE, EUA, 101, 409-412.

Oliveira, A. T. J. (2000). Uso de um equipamento elétrico de palheta em argilas do Recife. Dissertação de Mestrado em Engenharia Civi UFPE, Recife, Pernambuco, Brasil.

Oliveira, J. T. R. (2002). A Influência da qualidade da amostra no comportamento Tensão-Deformação-Resistência de argilas moles. Tese de Doutorado em Engenharia Civil, COPPE/UFRJ, Rio de Janeiro. 264p http://www.coc.ufrj.br/pt/teses-de-doutorado/146-2002/923-joaquim-teodoro-romao-de-oliveira.

Oliveira, J. T. R. (1991) Ensaios piezocones em um depósito de argila mole na cidade do Recife. Dissertação de Mestrado em Engenharia Civil, COPPE/UFRJ, Rio de Janeiro. 195p. https://pantheon.ufrj.br/bitstream/11422/4018/1/173229.pdf

Pacheco Silva, F. (195). Shearing strength of a soft clay deposit near Rio de Janeiro. Géotechnique. 3, 300 - 306.

Rocha, F. M. A. (2016). Compressibilidade de uma argila orgânica mole do bairro Chão de Estrelas no Recife. Dissertação Mestrado em Engenharia Civil Universidade Católica de Pernambuco, Recife, Pernambuco, Brasil. 127p. http://tede2.unicap.br:8080/bitstream/tede/70/1/felipe_melo_assis_rocha.pdf.

Sandroni. S.S., Brugger, P. J., Almeida, M.S.S \& Lacerda, W.A. (1997). Geotechnical properties of Sergipe clay. Proc. Int. Symp, Developments in Soil and Pavement Mechanic, , 271-276 Rio de Janeiro, Brazil.

Santos, A. A., Bridi L. Pires P. (2018) Caracterização da subsuperfície na transição entre Formação Bbarreiras e depósitos quaternários em Vitória - ES. Revista de Ciência, Tecnologia e Inovação, 4(7), 79-93. 
Research, Society and Development, v. 11, n. 3, e3711326123, 2022

(CC BY 4.0) | ISSN 2525-3409 | DOI: http://dx.doi.org/10.33448/rsd-v11i3.26123

Skempton, A. W., \& Northey, R. D. (1952). The sensitivity of clays. Géotechnique, 3(1), 30 - 53.

Schmertmann, J. H. (1953). Estimating the True Consolidation Behavior of Clay from Laboratory Test Results. Proceedings of the American Society of Civil Engineers, $79,1-26$.

Schmertmann, J. H. (1955). The Undisturbed Consolidation Behavior of Clay. Trans. Soc. Civ. Eng., 120, 1201 - 1233.

Soares, V. B. (1975) Propriedades de resistência de uma argila orgânica do Recife. . Dissertação (Mestrado em Ciências) - Universidade Federal da Paraíba, Campina Grande, Paraiba, Brasil. http://dspace.sti.ufcg.edu.br:8080/jspui/handle/riufcg/3821

Teixeira, D. C. L. (1972). Características geotécnicas dos depósitos de argila mole do Recife à influência da matéria orgânica. Dissertação de Mestrado em Engenharia Civil - COPPE/UFRJ, Rio de Janeiro. 94p. http://hdl.handle.net/11422/2303

Vieira, M. N. A., Alves, C. N., Ferreira, V. S. \& Barbosa, C. N. L. (2021). Modelagem de propriedades geológico-geotécnicas em solos de Belém do Pará. Research, Society and Development, 10 (2),p e40810212664, http://dx.doi.org/10.33448/rsd-v10i2.12664 